\title{
CORRIGENDUM
}

\section{Innovative education and training in high power laser plasmas (PowerLaPs) for plasma physics, high power laser matter interactions and high energy density physics: experimental diagnostics and simulations - CORRIGENDUM}

John Pasley ${ }^{\circledR 1}$, Georgia Andrianaki ${ }^{2}$, Jon Imanol Apiñaniz ${ }^{8,9}$, Andreas Baroutsos ${ }^{2}$, Dimitri Batani ${ }^{3}$, Emmanouil P. Benis ${ }^{4}$, Andrea Ciardi ${ }^{5,6}$, Donna Cook ${ }^{1}$, Massimo de $\mathrm{Marco}^{8,9}$, Vasilios Dimitriou ${ }^{2}$, Brendan Dromey ${ }^{7}$, Ioannis Fitilis ${ }^{2}$, Giancarlo Gatti ${ }^{8}$, Anastasios Grigoriadis ${ }^{4}$, Marine Huault ${ }^{8,9}$, Jose Antonio Pérez Hernández ${ }^{8}$, Evaggelos Kaselouris ${ }^{2}$, Ondrej Klimo ${ }^{10}$, Michel Koenig ${ }^{5}$, George Koundourakis ${ }^{2}$, Milan Kucharik ${ }^{10}$, Jiri Limpouch ${ }^{10}$, Richard Liska ${ }^{10}$, Carlos Salgado Lopez ${ }^{8,9}$, Sophia Malko $^{8,9}$, Susana Olmos-Migueláñez ${ }^{11,12}$, Yannis Orphanos ${ }^{2}$, Valeria Ospina ${ }^{8,9}$, Nektarios A. Papadogiannis ${ }^{2}$, Stelios Petrakis ${ }^{4}$, Jan Psikal ${ }^{10}$, Mauricio Rico ${ }^{8,9}$, Maria Serena Rivetta ${ }^{11}$, María-José Rodríguez-Conde ${ }^{11,12}$, João Jorge Santos ${ }^{3}$, Milan Sinor ${ }^{10}$, Alexandros Skoulakis ${ }^{2}$, Ioannis Tazes ${ }^{2}$, Laura Tejada Pascual $^{3}$, Michael Touati ${ }^{8,9}$, Calliope Tsitou $^{2}$, Pavel Vachal $^{10}$, Luca Volpe $^{8,13}$, Jiri Vyskocil ${ }^{10}$, Steven White ${ }^{7}$, Mark Yeung ${ }^{7}$, Ghassan Zeraouli ${ }^{8,9}$, and Michael Tatarakis ${ }^{2}$

${ }^{1}$ York Plasma Institute, University of York, Heslington, York YO10 5DQ, UK

${ }^{2}$ Hellenic Mediterranean University, Institute of Plasma Physics and Lasers - IPPL, 74100 Rethymnon, 73133 Chania, Crete, Greece

${ }^{3}$ University of Bordeaux, CNRS, CEA, CELIA (Centre Lasers Intenses et Applications), UMR 5107, F-33405 Talence, France

${ }^{4}$ Department of Physics, University of Ioannina, GR 45110 Ioannina, Greece

${ }^{5}$ LULI - CNRS, CEA, Sorbonne Universiés, Ecole Polytechnique, Institut Polytechnique de Paris - F-91128 Palaiseau Cedex, France

${ }^{6}$ Observatoire de Paris, 5 Place J Janssen, Meudon 92195, France

${ }^{7}$ Centre for Plasma Physics, School of Mathematics and Physics, Queen's University Belfast, Belfast BT7 1NN, UK

${ }^{8}$ Centro de Láseres Pulsados (CLPU), Edificio M5, Parque Cientfico, C/ Adaja 8, 37185 Villamayor, Salamanca, Spain

${ }^{9}$ University of Salamanca, 37008 Salamanca, Spain

${ }^{10}$ Faculty of Nuclear Sciences and Physical Engineering, Czech Technical University in Prague, Brehova 7, 115 19, Prague 1, Czech Republic

${ }^{11}$ University Institute for Educational Sciences, University of Salamanca, 37008 Salamanca, Spain

${ }^{12}$ Group of Research 'Interaction and e Learning' (GRIAL), University of Salamanca, 37008 Salamanca, Spain

${ }^{13}$ CLPU Laser-Plasma Chair at University of Salamanca, 37008 Salamanca, Spain

DOI: http://doi:10.1017/hpl.2020.4, Published by Cambridge University Press, 18 February 2020

The original publication omitted the following authors from the list of authors on the title page: Jon Imanol Apiñaniz, Massimo de Marco, Mauricio Rico and Michael Touati and mis-spelled the surname of Ghassan Zeraouli.

\section{Reference}

1. J. Pasley, et al., High Power Laser Sci. Eng. 8, e5 (2020).

Correspondence to: J. Pasley, York Plasma Institute, University of York, Heslington, York YO10 5DQ, UK. Email: john.pasley@york.ac.uk

(C) The Author(s) 2020. This is an Open Access article, distributed under the terms of the Creative Commons Attribution licence (http://creativecommons.org/ licenses/by/4.0/), which permits unrestricted re-use, distribution, and reproduction in any medium, provided the original work is properly cited. 